\title{
An Improved Version of Residual Power Series Method for Space- Time Fractional Problems
}

\author{
Mine Aylin Bayrak $\mathbb{D}^{1},{ }^{\text {Ali Demir }} \mathbb{D}^{1},{ }^{1}$ and Ebru Ozbilge $\mathbb{B D}^{2}$ \\ ${ }^{1}$ Department of Mathematics, Kocaeli University, Izmit, Kocaeli, Turkey \\ ${ }^{2}$ Department of Mathematics and Statistics, American University of the Middle East, Egaila, Kuwait \\ Correspondence should be addressed to Mine Aylin Bayrak; aylin@kocaeli.edu.tr
}

Received 28 September 2021; Accepted 29 December 2021; Published 15 January 2022

Academic Editor: Soheil Salahshour

Copyright (c) 2022 Mine Aylin Bayrak et al. This is an open access article distributed under the Creative Commons Attribution License, which permits unrestricted use, distribution, and reproduction in any medium, provided the original work is properly cited.

\begin{abstract}
The task of present research is to establish an enhanced version of residual power series (RPS) technique for the approximate solutions of linear and nonlinear space-time fractional problems with Dirichlet boundary conditions by introducing new parameter $\lambda$. The parameter $\lambda$ allows us to establish the best numerical solutions for space-time fractional differential equations (STFDE). Since each problem has different Dirichlet boundary conditions, the best choice of the parameter $\lambda$ depends on the problem. This is the major contribution of this research. The illustrated examples also show that the best approximate solutions of various problems are constructed for distinct values of parameter $\lambda$. Moreover, the efficiency and reliability of this technique are verified by the numerical examples.
\end{abstract}

\section{Introduction}

The well-established tool fractional differential equations (FDEs) attract growing attention of scientists in a wide range of scientific areas such as biology, physics, and engineering since it is very effective and accurate for modelling many processes in real life [1-11].

In FDEs, noninteger derivatives are taken into account to include memory of the system. Fractional mathematical models have results which are very close to the experimental data [12]. Since FDEs are applicable and variable, the progress of them is very rapid in diverse fields of science and engineering. Therefore, studies on solving FDEs produce efficient and reliable techniques to construct numerical and analytical solutions of them [13-20].

Diverse methods such as a semianalytical approach, a modified wavelet approach, linearized novel operational matrix-based scheme, hybrid spectral linearized scheme, finite difference/spectral algorithm, innovative operational matrix-based computational scheme, and a Chelyshkov polynomial-based algorithm are utilized to construct the solutions of FDEs [20-22].
RPS technique, which can be applied directly to the problem, is one of the most convenient ones to establish approximate solutions with high precision for linear and nonlinear FDEs [21-28]. Moreover, RPS technique allows us to construct the exact solution of the initial value problems whose solutions are polynomial.

Various mathematical models including differential equations such as model of vibration equation of large membranes, fractional Black-Scholes option pricing equations, and time-fractional fuzzy vibration equation of large membranes are analyzed by the RPS technique $[27,28]$.

Modified versions of the RPS technique are developed to acquire better approximate solutions of FDEs such as the solutions of STFDE in [29-34].

The motivation of this study is to employ a new version of RPS technique to establish better approximate solutions of fractional mathematical models including STFDE in Caputo sense. In the RPS technique, determining a suitable estimation for the initial guess approximations plays an important role. Therefore, in this study, we try to construct best initial guess approximation by introducing a new parameter. The novelty of this method is that new parameter $\lambda$ is introduced to get 
the best approximate solution for the fractional mathematical problems including STFDE with Dirichlet boundary conditions. When we are given Dirichlet boundary conditions, we utilize the parameter $\lambda$ to determine the initial rate of change which enables us to acquire the best approximate solution by the RPS technique.

\section{Preliminaries}

Now, the basic concepts of fractional calculus are presented [1-5].

Definition 1. The Riemann-Liouville (R-L) fractional derivative operator $D^{\alpha}$ of order $\alpha$ is described as $[2,5]$

$$
D^{\alpha} f(t)=\left\{\begin{array}{l}
\frac{1}{\Gamma(n-\alpha)} \frac{d}{d t^{n}} \int_{0}^{t} \frac{f(x)}{(t-x)^{\alpha-n+1}} d x, \quad n-1<\alpha<n, \\
\frac{\partial^{n}}{\partial t^{n}} f(t), \quad \alpha=n,
\end{array}\right.
$$

where $n \in Z^{+}$and $\alpha \in R^{+}$.

Definition 2. The R-L fractional-order integration operator $J^{\alpha}$ is described as $[2,5]$

$$
J^{\alpha} f(t)=\frac{1}{\Gamma(\alpha)} \int_{0}^{t}(t-x)^{\alpha-1} f(x) d x, \quad x>0, \alpha>0 .
$$

Following Podlubny [2], we may have

$$
\begin{aligned}
J^{\alpha} t^{n} & =\frac{\Gamma(n+1)}{\Gamma(n+\alpha+1} t^{n+\alpha}, \\
D^{\alpha} t^{n} & =\frac{\Gamma(n+1)}{\Gamma(n-\alpha+1} t^{n-\alpha} .
\end{aligned}
$$

Definition 3. The $\alpha^{\text {th }}$-order derivative of $f(t)$ in Caputo sense is defined as $[2,5]$

$$
{ }^{C} D^{\alpha} f(t)=\left\{\begin{array}{l}
\frac{1}{\Gamma(n-\alpha)} \int_{0}^{t} \frac{f^{n}(x)}{(t-x)^{\alpha-n+1}} d x, \quad n-1<\alpha<n, \\
\frac{\partial^{n} f(t)}{\partial t^{n}}, \quad \alpha=n .
\end{array}\right.
$$

Definition 4 (see $[2,5])$.

(1) $D^{\alpha} J^{\alpha} f(t)=f(t)$

(2) $J^{\alpha} D^{\alpha} f(t)=f(t)-\sum_{k=0}^{n} f^{(k)}\left(0^{+}\right)\left(t^{k} / k !\right), t>0$
TABLE 1: The approximate values of $u_{31}(x, t)$ for various values of $\lambda$ at $\alpha=1$ and $\beta=2$.

\begin{tabular}{cccccccc}
\hline$x$ & $t$ & Exact & $\lambda=-1$ & $\lambda=0$ & $\lambda=1$ & $\lambda=2$ & $\lambda=5$ \\
\hline & 0.3 & 0.21893 & 0.01731 & 0.10942 & 0.20153 & 0.29364 & 0.56997 \\
0.3 & 0.6 & 0.16218 & 0.01214 & 0.08038 & 0.14862 & 0.21686 & 0.42157 \\
& 0.9 & 0.12015 & 0.00611 & 0.05667 & 0.10722 & 0.15777 & 0.30942 \\
\hline & 0.3 & 0.41830 & 0.03307 & 0.20906 & 0.38505 & 0.56104 & 1.08902 \\
0.6 & 0.6 & 0.30988 & 0.02320 & 0.15358 & 0.28396 & 0.41434 & 0.80548 \\
& 0.9 & 0.22957 & 0.01168 & 0.10827 & 0.20486 & 0.30144 & 0.59120 \\
\hline & 0.3 & 0.58030 & 0.04587 & 0.29003 & 0.53418 & 0.77833 & 1.51080 \\
0.9 & 0.6 & 0.42990 & 0.03219 & 0.21306 & 0.39394 & 0.57481 & 1.11743 \\
& 0.9 & 0.31848 & 0.01621 & 0.15020 & 0.28420 & 0.41819 & 0.82017 \\
\hline
\end{tabular}

Definition 5. $(k, l)$-truncated series $u_{k l}(x, t)$ of the RPS method is given as

$u_{k l}(x, t)=\sum_{n=0}^{i-1} \frac{a_{n}(x)}{n !}+\sum_{n=1}^{k} \sum_{m=0}^{l} f_{n m}(x) \frac{t^{n \alpha+m}}{\Gamma(1+n \alpha+m)}, \quad n-1<\alpha \leq n, t>0$,

$u_{k l}(x, t)=\sum_{n=0}^{i-1} \frac{b_{n}(t)}{n !}+\lambda \sum_{n=1}^{k} \sum_{m=0}^{l}\left(g_{n m}(t)-g_{n m-1}(t)\right) \frac{x^{n \beta+m}}{\Gamma(1+n \beta+m)}, \quad n-1<\beta \leq n, x>0$.

\section{RPS Technique for Fractional Mathematical Models with Dirichlet Boundary Conditions}

We provide an efficient and reliable method for the solution of STFDE with Dirichlet boundary conditions. Let us take the following initial-boundary value problem into consideration:

$$
\begin{gathered}
D_{t}^{\alpha} u=D_{x}^{\beta} u+F(u), \\
u(x, 0)=f_{0}(x), \\
u(0, t)=g_{0}(t), \\
u(1, t)=g_{1}(t) .
\end{gathered}
$$

The $(k, 0)$-truncated series $u_{k 0}(x, t)$ is established by taking Equations (6) and (7) into account as follows:

$$
u_{k 0}(x, t)=f_{00}(x)+\sum_{n=1}^{k} f_{n 0}(x) \frac{t^{n \alpha}}{\Gamma(1+n \alpha)}, \quad t>0,0<\alpha \leq 1 .
$$

The use of Equations (1) and (3) leads to the kl-truncated series $u_{k l}(x, t)$ as follows:

$$
\begin{aligned}
u_{k l}(x, t)= & g_{00}(t)+\lambda\left(g_{01}(t)-g_{00}(t)\right) x+\lambda \sum_{n=1}^{k} \sum_{m=1}^{l}\left(g_{n m}(t)-g_{n m-1}(t)\right) \\
& \cdot \frac{x^{n \beta+m}}{\Gamma(1+n \beta+m)}, \quad x>0,1<\beta \leq 2 .
\end{aligned}
$$


TABLE 2: The approximate values of $u_{31}(x, t)$ with the parameter $\lambda=1.22$ and various orders $\alpha$ and $\beta$.

\begin{tabular}{|c|c|c|c|c|c|c|c|c|}
\hline$x$ & $t$ & Exact & $\begin{array}{l}\alpha=1 \\
\beta=2\end{array}$ & $\begin{array}{c}\alpha=1 \\
\beta=1.9\end{array}$ & $\begin{array}{c}\alpha=1 \\
\beta=1.8\end{array}$ & $\begin{array}{c}\alpha=0.9 \\
\beta=2\end{array}$ & $\begin{array}{l}\alpha=0.9 \\
\beta=1.9\end{array}$ & $\begin{array}{l}\alpha=0.9 \\
\beta=1.8\end{array}$ \\
\hline \multirow{3}{*}{0.3} & 0.3 & 0.21893 & 0.22179 & 0.22642 & 0.23048 & 0.21694 & 0.22205 & 0.22659 \\
\hline & 0.6 & 0.16218 & 0.16363 & 0.17129 & 0.17831 & 0.16014 & 0.16806 & 0.17524 \\
\hline & 0.9 & 0.12015 & 0.11834 & 0.12841 & 0.13752 & 0.11581 & 0.12663 & 0.13603 \\
\hline \multirow{3}{*}{0.6} & 0.3 & 0.41830 & 0.42377 & 0.42683 & 0.42944 & 0.41489 & 0.41853 & 0.42175 \\
\hline & 0.6 & 0.30988 & 0.31264 & 0.31944 & 0.32588 & 0.30551 & 0.31252 & 0.31909 \\
\hline & 0.9 & 0.22957 & 0.22611 & 0.23596 & 0.24531 & 0.22045 & 0.23117 & 0.24104 \\
\hline \multirow{3}{*}{0.9} & 0.3 & 0.58030 & 0.58789 & 0.58576 & 0.58351 & 0.57654 & 0.57492 & 0.57319 \\
\hline & 0.6 & 0.42990 & 0.43373 & 0.43516 & 0.43667 & 0.42266 & 0.42409 & 0.42558 \\
\hline & 0.9 & 0.31848 & 0.31368 & 0.31807 & 0.32267 & 0.30382 & 0.30869 & 0.31383 \\
\hline
\end{tabular}

An approximation for (6)-(8) is achieved by employing the new RPS technique as follows:

$$
\begin{aligned}
u(x, t)= & \frac{1}{2}\left(f_{00}(x)+g_{00}(t)+\lambda\left(g_{01}(t)-g_{00}(t)\right) x+\sum_{n=1}^{\infty} f_{n 0}(x) \frac{t^{n \alpha}}{\Gamma(1+n \alpha)}\right. \\
& \left.+\sum_{n=1}^{\infty} g_{n 0}(t) \frac{x^{n \beta}}{\Gamma(1+n \beta)}+\lambda \sum_{n=1}^{\infty}\left(g_{n 1}(t)-g_{n 0}(t)\right) \frac{x^{n \beta+1}}{\Gamma(2+n \beta)}\right) .
\end{aligned}
$$

Notice that we introduce a new parameter $\lambda$ in this approximate solutions to get a better approximation of the problems (6)-(8). Hence, the $(k, l)$-truncated series of $u_{k l}(x$ $, t)$ of $u(x, t)$ is achieved as follows:

$$
\begin{aligned}
u_{k l}(x, t)= & \frac{1}{2}\left(f_{00}(x)+g_{00}(t)+\lambda\left(g_{01}(t)-g_{00}(t)\right) x+\sum_{n=1}^{k} f_{n 0}(x) \frac{t^{n \alpha}}{\Gamma(1+n \alpha)}\right. \\
& \left.+\sum_{n=1}^{k} g_{n 0}(t) \frac{x^{n \beta}}{\Gamma(1+n \beta)}+\lambda \sum_{n=1}^{k} \sum_{m=1}^{l}\left(g_{n m}(t)-g_{n m-1}(t)\right) \frac{x^{n \beta+m}}{\Gamma(1+n \beta+m)}\right) .
\end{aligned}
$$

Equation (13) leads to the approximate solution $u_{00}(x, t)$ to become

$$
u_{00}(x, t)=\frac{1}{2}\left(f_{00}(x)+g_{00}(t)+\lambda\left(g_{01}(t)-g_{00}(t)\right) x\right) .
$$

The RPS technique leads to the following definition of the $k l$ residual function $\operatorname{Re} s_{k l}(x, t)$ as

$$
\operatorname{Re} s_{k l}(x, t)=D_{t}^{\alpha} u_{k l}-D_{x}^{\beta} u_{k l}-F\left(u_{k l}\right)
$$

In order to establish the coefficients $g_{i j}(t), i, j=1,2,3$, $\cdots, k$, plunge Equation (12) into Equation (14), and solve the following equation

$$
D_{x}^{(k-1) \beta} D_{x}^{l} \operatorname{Re} s_{k l}(0, t)=0 .
$$

To acquire $g_{10}(t)$, taking $k=1$ and $l=0$ in Equation (14)

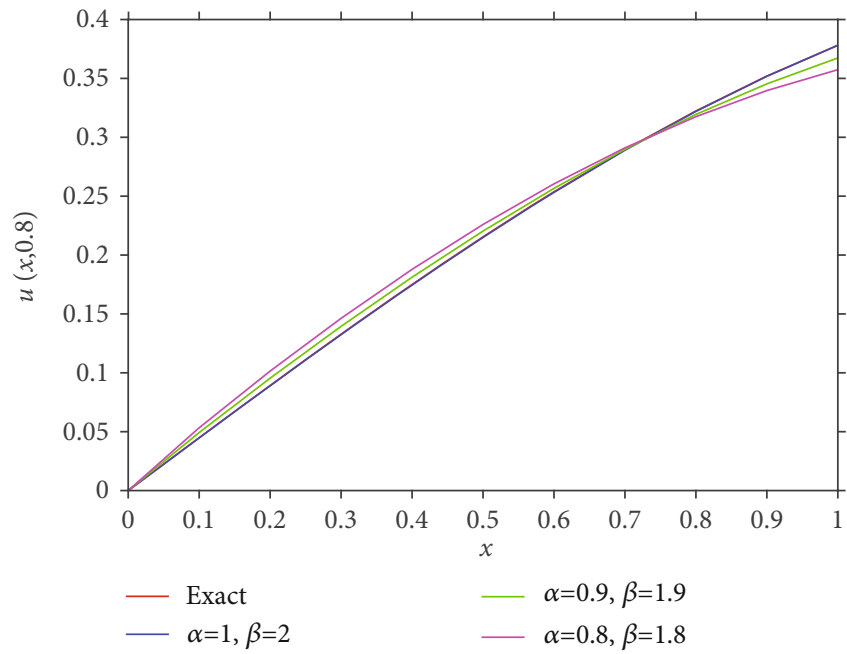

FIgURE 1: The graphics of approximate solution $u_{31}(x, t)$ at $t=0.8$ for $\lambda=1.22$ and various orders $\alpha$ and $\beta$.

leads to

$$
\begin{aligned}
\operatorname{Re} s_{10}(x, t)= & f_{10}(x)+D_{t}^{\alpha} g_{00}(t)+\lambda\left(D_{t}^{\alpha} g_{01}(t)-D_{t}^{\alpha} g_{00}(t)\right) x \\
& -D_{x}^{\beta} f_{00}(x)-D_{x}^{\beta} f_{10}(x) \frac{t^{\alpha}}{\Gamma(1+\alpha)}-g_{10}(t)-F\left(u_{10}\right),
\end{aligned}
$$

where

$$
\begin{aligned}
u_{10}(x, t)= & \frac{1}{2}\left(f_{00}(x)+g_{00}(t)+\lambda\left(g_{01}(t)-g_{00}(t)\right) x+f_{10}(x) \frac{t^{\alpha}}{\Gamma(1+\alpha)}\right. \\
& \left.+g_{10}(t) \frac{x^{\beta}}{\Gamma(1+\beta)}\right) .
\end{aligned}
$$

Employing Equation (15) allows us to have

$g_{10}(t)=f_{10}(0)+D_{t}^{\alpha} g_{00}(t)-D_{x}^{\beta} f_{00}(0)-D_{x}^{\beta} f_{10}(0) \frac{t^{\alpha}}{\Gamma(1+\alpha)}-F\left(u_{10}(0, t)\right)$ 
TABLE 3: The approximate values of $u_{21}(x, t)$ for various values of $\lambda$ at $\alpha=1$ and $\beta=2$.

\begin{tabular}{cccccccc}
\hline$x$ & $t$ & Exact & $\lambda=-1$ & $\lambda=0$ & $\lambda=1$ & $\lambda=2$ & -1 \\
0.3 & 0.3 & -0.53847 & -0.03648 & -0.26301 & -0.48954 & -0.71608 & -1.39567 \\
& 0.6 & -0.98116 & -0.01907 & -0.43146 & -0.84385 & -25624 & -2.15258 \\
\hline \multirow{3}{*}{0.6} & 0.9 & -1.78779 & 0.09664 & -0.65310 & -1.40284 & -4.40181 \\
\hline & 0.3 & -1.02885 & -0.06983 & -0.50253 & -0.93524 & -1.36794 \\
& 0.6 & -1.87468 & -0.03878 & -0.82438 & -1.60998 & -2.39558 & -4.66605 \\
& 0.9 & -3.41589 & 0.17085 & -1.24786 & -2.66657 & -4.08528 & -8.34142 \\
\hline \multirow{3}{*}{0.9} & 0.3 & -1.42731 & -0.09709 & -0.69716 & -1.29723 & -1.89730 & -3.69751 \\
& 0.6 & -2.60074 & -0.05891 & -1.14366 & -2.22840 & -3.31315 & -6.56740 \\
& 0.9 & -4.73885 & 0.20655 & -1.73115 & -3.66885 & -5.60655 & -11.41964 \\
\hline
\end{tabular}

TABLE 4: The approximate values of $u_{21}(x, t)$ with the parameter $\lambda=1.47$ for various orders $\alpha$ and $\beta$.

\begin{tabular}{ccccccccc}
\hline$x$ & $t$ & \multirow{2}{*}{ Exact } & $\alpha=1$ & $\alpha=1$ & $\alpha=1$ & $\alpha=0.9$ & $\alpha=0.9$ & $\alpha=0.9$ \\
$\beta=2$ & $\beta=1.9$ & $\beta=1.8$ & $\beta=2$ & -0.64718 \\
\hline \multirow{3}{*}{0.3} & 0.3 & -0.53847 & -0.59601 & -0.60551 & -0.61355 & -0.62399 & -0.63650 & -1.9 \\
& 0.6 & -0.98116 & -1.03768 & -1.06509 & -1.08905 & -1.08716 & -1.12080 & -1.15039 \\
& 0.9 & -1.78779 & -1.75522 & -1.80729 & -1.85298 & -1.81910 & -1.87986 & -1.93342 \\
\hline \multirow{3}{*}{0.6} & 0.3 & -1.02885 & -1.13861 & -1.14310 & -1.14629 & -1.18552 & -1.19207 & -1.19710 \\
& 0.6 & -1.87468 & -1.97921 & -1.99778 & -2.01391 & -2.06547 & -2.08925 & -2.11030 \\
& 0.9 & -3.41589 & -3.33337 & -3.36702 & -3.39611 & -3.44429 & -3.48526 & -3.52129 \\
\hline \multirow{3}{*}{0.9} & 0.3 & -1.42731 & -1.57926 & -1.56996 & -1.56013 & -1.62855 & -1.61841 & -1.60792 \\
& 0.6 & -2.60074 & -2.73824 & -2.72758 & -2.71682 & -2.83788 & -2.82787 & -2.81811 \\
& 0.9 & -4.73885 & -4.57957 & -4.55689 & -4.53486 & -4.70650 & -4.68487 & -4.66435 \\
\hline
\end{tabular}

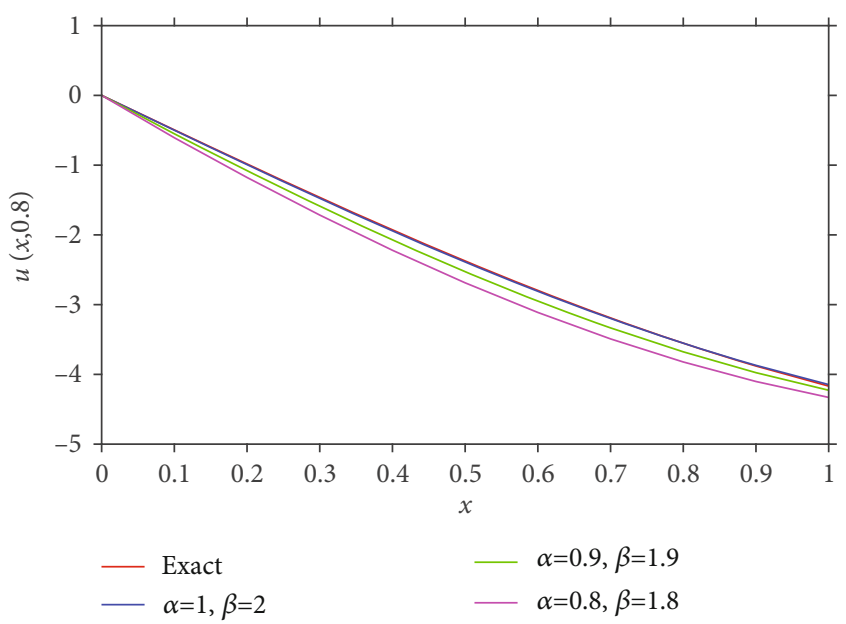

Figure 2: The graphics of approximate solution $u_{21}(x, t)$ at $t=0.8$ and $\lambda=1.47$ for various orders $\alpha$ and $\beta$.

Similarly, we take $k=2$ and $l=0$ in Equation (14) to establish the coefficient $g_{20}(t)$,

$$
\operatorname{Re} s_{20}(x, t)=D_{t}^{\alpha} u_{20}-D_{x}^{\beta} u_{20}-F\left(u_{20}\right) \text {, }
$$

where

$$
\begin{aligned}
u_{20}(x, t)= & \frac{1}{2}\left(f_{00}(x)+g_{00}(t)+\lambda\left(g_{01}(t)-g_{00}(t)\right) x+f_{10}(x) \frac{t^{\alpha}}{\Gamma(1+\alpha)}\right. \\
& \left.+f_{20}(x) \frac{t^{2 \alpha}}{\Gamma(1+2 \alpha)}+g_{10}(t) \frac{x^{\beta}}{\Gamma(1+\beta)}+g_{20}(t) \frac{x^{2 \beta}}{\Gamma(1+2 \beta)}\right)
\end{aligned}
$$

Taking Equation (15) into account leads to

$$
\begin{aligned}
g_{20}(t)= & D_{x}^{\beta} f_{10}(0)+D_{x}^{\beta} f_{20}(0) \frac{t^{\alpha}}{\Gamma(1+\alpha)}+D_{t}^{\alpha} g_{10}-D_{x} D_{x}^{\beta} f_{00}(0) \\
& -D_{x} D_{x}^{\beta} f_{10}(0) \frac{t^{\alpha}}{\Gamma(1+\alpha)}-D_{x} D_{x}^{\beta} f_{20}(0) \frac{t^{2 \alpha}}{\Gamma(1+2 \alpha)} \\
& -D_{x}^{\beta} F\left(u_{20}(0, t)\right) .
\end{aligned}
$$

As in the previous steps, taking $k=1$ and $l=1$ in Equation (14) leads to

$$
\operatorname{Re} s_{11}(x, t)=D_{t}^{\alpha} u_{11}-D_{x}^{\beta} u_{11}-F\left(u_{11}\right)
$$


TABLE 5: The approximate values of $u_{21}(x, t)$ for various values of $\lambda$ at $\alpha=1$ and $\beta=2$.

\begin{tabular}{cccccccc}
\hline$x$ & $t$ & Exact & $\lambda=-1$ & $\lambda=0$ & $\lambda=1$ & $\lambda=2$ & $\lambda=5$ \\
\hline \multirow{4}{*}{0.1} & 0.1 & 0.25000 & -0.00069 & 0.12385 & 0.24736 & 0.36982 & 0.73096 \\
& 0.2 & 0.33333 & -0.01045 & 0.15568 & 0.31996 & 0.48238 & 0.95855 \\
& 0.3 & 0.50000 & -0.05424 & 0.19548 & 0.44103 & 0.68242 & 1.38158 \\
& 0.4 & 1.00000 & -0.26021 & 0.24325 & 0.73005 & 1.20018 & 2.51057 \\
\hline \multirow{4}{*}{0.2} & 0.1 & 0.50000 & 0.00045 & 0.24683 & 0.48487 & 0.71458 & 1.35370 \\
& 0.2 & 0.66667 & -0.01960 & 0.30944 & 0.62366 & 0.92307 & 1.73240 \\
& 0.3 & 1.00000 & -0.10992 & 0.38784 & 0.85226 & 1.28336 & 2.37663 \\
& 0.4 & 2.00000 & -0.54568 & 0.48203 & 1.37640 & 2.13744 & 3.62055 \\
\hline \multirow{4}{*}{0.3} & 0.1 & 0.75000 & 0.00527 & 0.36804 & 0.70268 & 1.00920 & 1.75999 \\
& 0.2 & 1.00000 & -0.02614 & 0.45936 & 0.89486 & 1.28036 & 2.13685 \\
& 0.3 & 1.50000 & -0.16847 & 0.57396 & 1.20389 & 1.72132 & 2.59862 \\
& 0.4 & 3.00000 & -0.88166 & 0.71184 & 1.85534 & 2.54885 & 1.92936 \\
\hline & 0.1 & 1.00000 & 0.01561 & 0.48661 & 0.89095 & 1.22861 & 1.84161 \\
& 0.2 & 1.33333 & -0.02877 & 0.60352 & 1.11729 & 1.51255 & 1.98720 \\
0.4 & 0.3 & 2.00000 & -0.23134 & 0.75072 & 1.46612 & 1.91484 & 1.66103 \\
& 0.4 & 4.00000 & -1.29343 & 0.92821 & 2.08319 & 2.17149 & -3.96359 \\
\hline
\end{tabular}

where

$$
\begin{aligned}
u_{11}(x, t)= & \frac{1}{2}\left(f_{00}(x)+g_{00}(t)+\lambda\left(g_{01}(t)-g_{00}(t)\right) x+f_{10}(x) \frac{t^{\alpha}}{\Gamma(1+\alpha)}\right. \\
& \left.+g_{10}(t) \frac{x^{\beta}}{\Gamma(1+\beta)}+\lambda\left(g_{11}(t)-g_{10}(t)\right) \frac{x^{\beta+1}}{\Gamma(2+\beta)}\right) .
\end{aligned}
$$

Using Equation (15) when $k=1$ and $l=1$, we get

$$
\begin{aligned}
g_{11}(t)= & g_{10}(t)+D_{t}^{\alpha} g_{01}-D_{t}^{\alpha} g_{00}+\frac{1}{\lambda}\left(D_{x} f_{10}(0)-D_{x} D_{x}^{\beta} f_{00}(0)\right. \\
& \left.-D_{x} D_{x}^{\beta} f_{10}(0) \frac{t^{\alpha}}{\Gamma(1+\alpha)}-D_{x} F\left(u_{11}(0, t)\right)\right)
\end{aligned}
$$

To determine $g_{21}(t)$, substituting $k=2$ and $l=1$ into Equation (14), then

$$
\operatorname{Re} s_{21}(x, t)=D_{t}^{\alpha} u_{21}-D_{x}^{\beta} u_{21}-F\left(u_{21}\right) \text {, }
$$

where

$$
\begin{aligned}
u_{21}(x, t)= & \frac{1}{2}\left(f_{00}(x)+g_{00}(t)+\lambda\left(g_{01}(t)-g_{00}(t)\right) x+f_{10}(x) \frac{t^{\alpha}}{\Gamma(1+\alpha)}\right. \\
& +f_{20}(x) \frac{t^{2 \alpha}}{\Gamma(1+2 \alpha)}+g_{10}(t) \frac{x^{\beta}}{\Gamma(1+\beta)}+g_{20}(t) \frac{x^{2 \beta}}{\Gamma(1+2 \beta)} \\
& \left.+\lambda\left(g_{11}(t)-g_{10}(t)\right) \frac{x^{\beta+1}}{\Gamma(2+\beta)}+\lambda\left(g_{21}(t)-g_{20}(t)\right) \frac{x^{2 \beta+1}}{\Gamma(2+2 \beta)}\right) .
\end{aligned}
$$

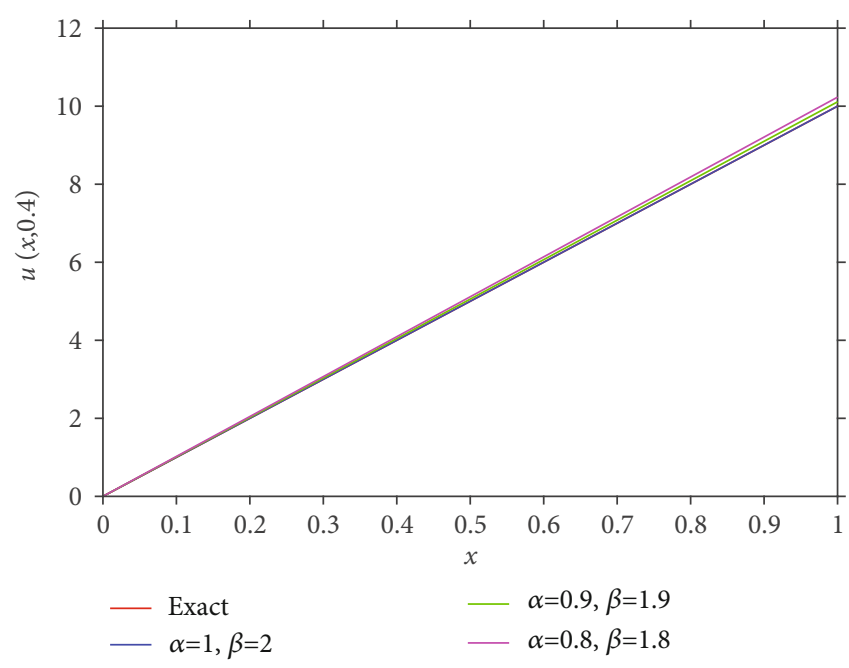

FIgURE 3: The graphics of approximate solution $u_{21}(x, t)$ at $t=0.8$ and $\lambda=1.16$ for various orders $\alpha$ and $\beta$.

Using Equation (15) when $k=2$ and $l=1$, we get

$$
\begin{aligned}
g_{21}(t)= & g_{20}(t)+D_{t}^{\alpha} g_{11}-D_{t}^{\alpha} g_{10}+\frac{1}{\lambda}\left(D_{x}^{\beta} D_{x} f_{10}(0)-D_{x}^{\beta} D_{x} f_{20}(0) \frac{t^{\alpha}}{\Gamma(1+\alpha)}\right. \\
& -D_{x}^{\beta} D_{x} D_{x}^{\beta} f_{00}(0)-D_{x}^{\beta} D_{x} D_{x}^{\beta} f_{10}(0) \frac{t^{\alpha}}{\Gamma(1+\alpha)}-D_{x}^{\beta} D_{x} D_{x}^{\beta} f_{20}(0) \frac{t^{2 \alpha}}{\Gamma(1+2 \alpha)} \\
& \left.-D_{x}^{\beta} D_{x} F\left(u_{21}(0, t)\right)\right),
\end{aligned}
$$

and so on.

Now, the next step is to obtain the coefficients $f_{i 0}(x), i$ $=1,2,3, \cdots, k$, by Equation (9).

The equation

$$
D_{t}^{(k-1) \alpha} \operatorname{Re} s_{k 0}(x, 0)=0
$$

allows us to establish the required coefficients $f_{i j}(x), i, j=1$ $, 2,3, \cdots, k$. Taking into $k=1$ in Equation (14) leads to

$$
\operatorname{Re} s_{10}(x, t)=D_{t}^{\alpha} u_{10}-D_{x}^{\beta} u_{10}-F\left(u_{10}\right)
$$

where

$$
u_{10}(x, t)=f_{00}(x)+f_{10}(x) \frac{t^{\alpha}}{\Gamma(1+\alpha)},
$$

which allows us to determine the coefficients $f_{10}(x)$ as follows:

$$
f_{10}(x)=D_{x}^{\beta} f_{00}(x)+F\left(u_{10}(x, 0)\right) .
$$

In a similar form, $f_{20}(x)$ is acquired by substituting $k=2$ into Equation (14) then

$$
\operatorname{Re} s_{20}(x, t)=D_{t}^{\alpha} u_{20}-D_{x}^{\beta} u_{20}-F\left(u_{20}\right) \text {, }
$$


TABLE 6: The approximate values of $u_{21}(x, t)$ with the parameter $\lambda=1.16$ for various orders $\alpha$ and $\beta$.

\begin{tabular}{|c|c|c|c|c|c|c|c|c|}
\hline$x$ & $t$ & Exact & $\begin{array}{l}\alpha=1 \\
\beta=2\end{array}$ & $\begin{array}{c}\alpha=1 \\
\beta=1.9\end{array}$ & $\begin{array}{c}\alpha=1 \\
\beta=1.8\end{array}$ & $\begin{array}{c}\alpha=0.9 \\
\beta=2\end{array}$ & $\begin{array}{l}\alpha=0.9 \\
\beta=1.9\end{array}$ & $\begin{array}{l}\alpha=0.9 \\
\beta=1.8\end{array}$ \\
\hline \multirow{4}{*}{0.1} & 0.1 & 0.25000 & 0.26702 & 0.26618 & 0.26499 & 0.27667 & 0.27579 & 0.27454 \\
\hline & 0.2 & 0.33333 & 0.34607 & 0.34468 & 0.34272 & 0.36510 & 0.36365 & 0.36160 \\
\hline & 0.3 & 0.50000 & 0.47993 & 0.47735 & 0.47371 & 0.50879 & 0.50614 & 0.50241 \\
\hline & 0.4 & 1.00000 & 0.80639 & 0.79890 & 0.78833 & 0.84529 & 0.83781 & 0.82726 \\
\hline \multirow{4}{*}{0.2} & 0.1 & 0.50000 & 0.52218 & 0.51696 & 0.51010 & 0.54089 & 0.53541 & 0.52822 \\
\hline & 0.2 & 0.66667 & 0.67256 & 0.66395 & 0.65263 & 0.70971 & 0.70069 & 0.68884 \\
\hline & 0.3 & 1.00000 & 0.92348 & 0.90747 & 0.88643 & 0.98027 & 0.96386 & 0.94229 \\
\hline & 0.4 & 2.00000 & 1.50712 & 1.46065 & 1.39956 & 1.58512 & 1.53873 & 1.47776 \\
\hline \multirow{4}{*}{0.3} & 0.1 & 0.75000 & 0.75361 & 0.73882 & 0.72018 & 0.78022 & 0.76470 & 0.74514 \\
\hline & 0.2 & 1.00000 & 0.95990 & 0.93549 & 0.90473 & 1.01331 & 0.98774 & 0.95554 \\
\hline & 0.3 & 1.50000 & 1.29424 & 1.24887 & 1.19170 & 1.37713 & 1.33062 & 1.27201 \\
\hline & 0.4 & 3.00000 & 1.99655 & 1.86481 & 1.69883 & 2.11404 & 1.98255 & 1.81688 \\
\hline \multirow{4}{*}{0.4} & 0.1 & 1.00000 & 0.94945 & 0.91897 & 0.88172 & 0.98220 & 0.95022 & 0.91114 \\
\hline & 0.2 & 1.33333 & 1.18850 & 1.13820 & 1.07672 & 1.25540 & 1.20273 & 1.13835 \\
\hline & 0.3 & 2.00000 & 1.55583 & 1.46234 & 1.34806 & 1.66207 & 1.56622 & 1.44907 \\
\hline & 0.4 & 4.00000 & 2.16900 & 1.89754 & 1.56576 & 2.32658 & 2.05563 & 1.72447 \\
\hline
\end{tabular}

where

$$
u_{20}(x, t)=f_{00}(x)+f_{10}(x) \frac{t^{\alpha}}{\Gamma(1+\alpha)}+f_{20}(x) \frac{t^{2 \alpha}}{\Gamma(1+2 \alpha)}
$$
form:

Hence, we have the coefficient $f_{20}(x)$ in the following

$$
f_{20}(x)=D_{x}^{\beta} f_{10}(x)+D_{t}^{\alpha} F\left(u_{20}(x, 0)\right)
$$

and so on. By substituting the coefficients $f_{i j}(x), i, j=1,2,3$ $, \cdots, k$, into Equations (18), (21), (24), and (27) lead to the coefficients $g_{i j}(t), i, j=1,2,3, \cdots, k$.

As a verification of the new RPS technique, we observe that we obtain as a classical exact solution of the problems (6)-(8) with integer order as $\beta \longrightarrow 2$ and $\alpha \longrightarrow 1$.

\section{Convergence Analysis}

Theorem 6. Let $u(x, t), D_{t}^{k \alpha} u(x, t) \in C\left[R_{1}, t_{0}\right] \times\left[R_{1}, t_{0}+R_{2}\right]$, and $D_{x}^{k \beta} u(x, t) \in C\left[x_{0}, R_{2}\right] \times\left[x_{0}+R_{2}, R_{2}\right]$, where $k=0,1,2$, $\cdots, p_{1}+1, j=0,1,2, \cdots, n-1$, and $l=0,1,2, \cdots, p_{2}+1, i=0$, $1,2, \cdots, m-1$. Moreover, $D_{t}^{k \alpha} u(x, t)$ can be differentiated $n$ -1 times with respect to ' $t$ ' on $\left(t_{0}, t_{0}+R_{1}\right)$ and $D_{x}^{l \beta} u(x, t)$ can be differentiated $m-1$ times with respect to ' $x$ ' on $\left(x_{0}\right.$, $\left.x_{0}+R_{2}\right)$. Then,

$$
u(x, t) \cong \frac{1}{2} \sum_{j=0}^{n-1} \sum_{i=0}^{p_{1}} F_{j+i \alpha}(x)\left(t-t_{0}\right)^{j+i \alpha}+\frac{\lambda}{2} \sum_{j=0}^{m-1} \sum_{i=0}^{p_{2}} G_{j+i \beta}(t)\left(x-x_{0}\right)^{j+i \beta},
$$

where $F_{j+i \alpha}(x)=\left(D_{t}^{j+i \alpha} /(\Gamma(j+i \alpha+1)) \Gamma(j+i \alpha+1)\right) u\left(x, t_{0}\right)$ and $G_{j+i \beta}(t)=\left(D_{x}^{j+i \beta} /(\Gamma(j+i \beta+1)) \Gamma(j+i \beta+1)\right) u\left(x_{0}, t\right)$.

Moreover, for $\exists$ values $\varepsilon_{1}$ and $\varepsilon_{2}$ such that $0 \leq \varepsilon_{1} \leq t$ and 0 $\leq \varepsilon_{2} \leq x$, the error term has the term as follows:

$$
\begin{aligned}
\left\|E_{p}(x, t)\right\| \leq & \frac{1}{2}\left[\sup _{t \in[0, T]}\left|\sum_{j=0}^{n-1}\left(\frac{D_{t}^{j+\left(p_{1}+1\right) \alpha}}{\Gamma\left(j+\left(p_{1}+1\right) \alpha+1\right)} u\left(x, \varepsilon_{1}\right) t^{j+\left(p_{1}+1\right) \alpha}\right)\right|\right. \\
& \left.+\inf _{\lambda \in[1,2]} \sup _{x \in[0, L]}\left|\lambda \sum_{j=0}^{m-1}\left(\frac{D_{x}^{j+\left(p_{2}+1\right) \beta}}{\Gamma\left(j+\left(p_{2}+1\right) \beta+1\right)} u\left(\varepsilon_{2}, t\right) x^{j+\left(p_{2}+1\right) \beta}\right)\right|\right] .
\end{aligned}
$$

Proof. The proof of this theorem follows from the proof 4.3 [27].

\section{Numerical Examples}

In this section, we provide illustrative examples to prove how effective and reliable the RPS technique proposed in this study is.

Example 1. Consider the STFDE with initial-boundary conditions

$$
\begin{gathered}
D_{t}^{\alpha} u=D_{x}^{\beta} u, \\
u(x, 0)=\sin (x), \\
u(0, t)=0, \\
u(1, t)=\exp (-t) \sin (1),
\end{gathered}
$$


for which

$$
u(x, t)=\exp (-t) \sin (x)
$$

represents the exact solution for $\alpha=1$ and $\beta=2$. Based on the RPS technique and above problem, we have the following coefficients:

$$
\begin{gathered}
f_{00}(x)=\sin (x), \\
g_{00}(t)=0, \\
g_{01}(t)=\exp (-t) \sin (1), \\
f_{10}(x)=\frac{1}{2 i}\left(\sum_{k=0}^{\infty} i^{k+2} \frac{x^{k+2-\beta}}{\Gamma(k+3-\beta)}-\sum_{k=0}^{\infty}(i)^{k+2} \frac{x^{k+2-\beta}}{\Gamma(k+3-\beta)}\right), \\
g_{10}(t)=0, \\
g_{11}(t)=\sin (1)\left(\sum_{k=0}^{\infty}(-1)^{k+1} \frac{t^{k+1-\alpha}}{\Gamma(k+2-\alpha)}\right), \\
\frac{1}{2 i}\left(\sum_{k=0}^{\infty} i^{k+4} \frac{x^{k+4-2 \beta}}{\Gamma(k+5-2 \beta)}-\sum_{k=0}^{\infty}(-i)^{k+4} \frac{x^{k+4-2 \beta}}{\Gamma(k+5-2 \beta)}\right), \\
g_{21}(t)=0, \quad \sin (1)\left(\sum_{k=0}^{\infty}(-1)^{k+2} \frac{t^{k+2-2 \alpha}}{\Gamma(k+3-2 \alpha)}\right), \\
f_{30}(x)=\frac{1}{2 i}\left(\sum_{k=0}^{\infty} i^{k+6} \frac{x^{k+6-3 \beta}}{\Gamma(k+7-3 \beta)}-\sum_{k=0}^{\infty}(-i)^{k+6} \frac{x^{k+6-3 \beta}}{\Gamma(k+7-3 \beta)}\right), \\
g_{30}(t)=0, \\
g_{31}(t)=\sin (1)\left(\sum_{k=0}^{\infty}(-1)^{k+3} \frac{t^{k+3-3 \alpha}}{\Gamma(k+4-3 \alpha)}\right) .
\end{gathered}
$$

In Table 1, the approximate solutions are given for various values of $\lambda$ with $\alpha=1$ and $\beta=2$. Table 2 provides the values of exact solution and an approximate solution $u_{31}(x$ ,t) for the parameter $\lambda=1.22$ and various orders of $\alpha$ and $\beta$. Based on Table 1, we conclude that approximate solutions have a higher accuracy as adding more components. Moreover, Figure 1 supports our conclusion.

Example 2. Take the initial-boundary value problem including STFDE with source function into consideration

$$
\begin{gathered}
D_{t}^{\alpha} u=D_{x}^{\beta} u+3 u \\
u(x, 0)=\cos \left(x+\frac{\pi}{2}\right), \\
u(0, t)=0, u(1, t)=\exp (2 t) \cos \left(1+\frac{\pi}{2}\right),
\end{gathered}
$$

for which

$$
u(x, t)=\exp (2 t) \cos \left(x+\frac{\pi}{2}\right)
$$

denotes the exact solution for $\alpha=1$ and $\beta=2$. Based on the
RPS technique and above problem, the following coefficients are obtained:

$$
\begin{aligned}
& f_{00}(x)=\cos \left(x+\frac{\pi}{2}\right) \\
& g_{00}(t)=0, \\
& g_{01}(t)=\exp (2 t) \cos \left(1+\frac{\pi}{2}\right), \\
& f_{10}(x)=\frac{1}{2 i}\left(\sum_{k=0}^{\infty}(-i)^{k+2} \frac{x^{k+2-\beta}}{\Gamma(k+3-\beta)}-\sum_{k=0}^{\infty} i^{k+2} \frac{x^{k+2-\beta}}{\Gamma(k+3-\beta)}\right) \\
& +3 \cos \left(x+\frac{\pi}{2}\right) \text {, } \\
& g_{10}(t)=0, \\
& g_{11}(t)=-3 \cos \left(1+\frac{\pi}{2}\right) \exp (2 t)+\cos \left(1+\frac{\pi}{2}\right) \sum_{k=0}^{\infty} 2^{k+1} \frac{t^{k+1-\alpha}}{\Gamma(k+2-\alpha)}, \\
& f_{20}(x)=\frac{1}{2 i}\left(\sum_{k=0}^{\infty} i^{k+2} \frac{x^{k+4-2 \beta}}{\Gamma(k+5-2 \beta)}-\sum_{k=0}^{\infty}(-i)^{k+2} \frac{x^{k+4-2 \beta}}{\Gamma(k+5-2 \beta)}\right) \\
& +\frac{6}{2 i}\left(\sum_{k=0}^{\infty}(-i)^{k+2} \frac{\mathrm{x}^{k+2-\beta}}{\Gamma(k+3-\beta)}-\sum_{k=0}^{\infty} i^{k+2} \frac{x^{k+2-\beta}}{\Gamma(k+3-\beta)}\right) \\
& +3 \cos \left(x+\frac{\pi}{2}\right)+9 \cos \left(x+\frac{\pi}{2}\right) \text {, } \\
& g_{20}(t)=0, \\
& g_{21}(t)=9 \cos \left(1+\frac{\pi}{2}\right) \exp (2 t)-6 \cos \left(1+\frac{\pi}{2}\right) \sum_{k=0}^{\infty} 2^{k+1} \frac{t^{k+1-\alpha}}{\Gamma(k+2-\alpha)} \\
& +\cos \left(1+\frac{\pi}{2}\right) \sum_{k=0}^{\infty} 2^{k+2} \frac{t^{k+2-2 \alpha}}{\Gamma(k+3-2 \alpha)} .
\end{aligned}
$$

In Table 3, the approximate solution is given for various values of $\lambda$ with $\alpha=1$ and $\beta=2$. Table 4 provides the values of exact solution and the approximation $u_{21}(x, t)$ with the parameter $\lambda=1.47$ and various orders $\alpha$ and $\beta$. Based on Table 3, we conclude that the approximate solution has a higher accuracy as adding more components. Moreover, Figure 2 confirms our conclusion.

Example 3. Consider the initial-boundary value problem including fractional Burger equation

$$
\begin{gathered}
D_{t}^{\alpha} u=D_{x}^{\beta} u+u u_{x}, \\
u(x, 0)=2 x, \\
u(0, t)=0, u(1, t)=\frac{2}{1-2 t},
\end{gathered}
$$


for which

$$
u(x, t)=\frac{2 x}{1-2 t}
$$

represents the exact solution for integer order derivatives. Based on the RPS technique and above problem, the following coefficients are established:

$$
\begin{gathered}
f_{00}(x)=2 x, \\
g_{00}(t)=0, \\
g_{01}(t)=\frac{2}{1-2 t}, \\
f_{10}(x)=4 x, \\
g_{10}(t)=0, \\
f_{20}(x)=16 x \\
g_{20}(t)=0 .
\end{gathered}
$$

In Table 5, the approximate solution is given for various values of $\lambda$ with $\alpha=1$ and $\beta=2$. Table 6 provides the values of exact solution and the approximation $u_{21}(x, t)$ with the parameter $\lambda=1.16$ and various orders $\alpha$ and $\beta$. Based on Table 5, we conclude that the approximate solution has a higher accuracy as adding more components. Moreover, our conclusion is supported by Figure 3.

\section{Conclusion}

In this study, the enhanced version of RPS technique is proposed to establish better approximate solutions of linear and nonlinear space-time fractional problems with Dirichlet boundary conditions by introducing new parameter $\lambda$. The illustrated examples present that the best approximate solutions are achieved for specific values of the parameter $\lambda$. Moreover, the value of parameter $\lambda$ which leads to the best approximate solution depends on the fractional mathematical problem. The numerical examples also prove that this new effective and reliable technique is easy to implement.

\section{Data Availability}

There is no data available.

\section{Conflicts of Interest}

The authors declare that they have no conflicts of interest.

\section{References}

[1] A. A. Kilbas, H. M. Srivastava, and J. J. Trujillo, Theory and Applications of Fractional Differential Equations, Elsevier, Amsterdam, 2006.

[2] I. Podlubny, Fractional Differential Equation, CA, Academic Press, San Diego, 1999.

[3] J. Sabatier, O. P. Agarwal, and J. A. T. Machado, Eds., Advances in Fractional Calculus: Theoretical Developments and Applications in Physics and Engineering, Springer, Dordrecht, 2007.
[4] S. G. Samko, A. A. Kilbas, and O. I. Marichev, Fractional Integrals and Derivatives Theory and Applications, Gordon and Breach, Amsterdam, 1993.

[5] K. S. Miller and B. Ross, An Introduction to the Fractional Calculus and Fractional Differential Equations, A Wiley Interscience Publication, John Wiley and Sons, New York, Chichester, Brisbane, Toronto and Singapore, 1993.

[6] S. Momani and Z. Odibat, "Analytical approach to linear fractional partial differential equations arising in fluid mechanics," Physics Letters A, vol. 355, no. 4-5, pp. 271-279, 2006.

[7] K. Seki, M. Wojcik, and M. Tachiya, "Fractional reactiondiffusion equation," The Journal of Chemical Physics, vol. 119, no. 4, pp. 2165-2170, 2003.

[8] R. K. Saxena, A. M. Mathai, and H. J. Haubold, "Fractional reaction-diffusion equations," Astrophysics and Space Science, vol. 305, no. 3, pp. 289-296, 2006.

[9] M. O. Vlad and J. Ross, "Systematic derivation of reactiondiffusion equations with distributed delays and relations to fractional reaction diffusion equations and hyperbolic transport equations: application to the theory of Neolithic transition," Physical Review E, vol. 66, p. 12, 2002.

[10] H. Weitzner and G. M. Zaslavsky, "Some applications of fractional equations," Communications in Nonlinear Science and Numerical Simulation, vol. 8, no. 3-4, pp. 273-281, 2003.

[11] A. A. M. Arafa, S. Z. Rida, A. A. Mohammadein, and H. M. Ali, "Solving nonlinear fractional differential equation by generalized Mittag-Leffler function method," Communications in Theoretical Physics, vol. 59, no. 6, pp. 661-663, 2013.

[12] A. A. M. Arafa, S. Z. Rida, and M. Khalil, “A fractional-order model of HIV infection: numerical solution and comparisons with data of patients," International Journal of Biomathematics, vol. 7, no. 4, article 1450036, 2014.

[13] M. Alquran, M. Ali, M. Alsukhour, and I. Jaradat, "Promoted residual power series technique with Laplace transform to solve some time-fractional problems arising in physics," Results in Physics, vol. 19, article 103667, 2020.

[14] S. Momani and Z. Odibat, "Analytical solution of a timefractional Navier-Stokes equation by Adomian decomposition method," Applied Mathematics and Computation, vol. 177, no. 2, pp. 488-494, 2006.

[15] S. Odibat and S. Momani, "Application of variational iteration method to nonlinear differential equations of fractional order," International Journal of Nonlinear Sciences and Numerical Simulation, vol. 7, no. 1, pp. 27-34, 2006.

[16] X. J. Yang and D. Baleanu, "Fractal heat conduction problem solved by local fractional variation iteration method," Thermal Science, vol. 17, no. 2, pp. 625-628, 2013.

[17] X. Zhang, J. Zhao, J. Liu, and B. Tang, "Homotopy perturbation method for two dimensional time-fractional wave equation," Applied Mathematical Modelling, vol. 38, no. 23, pp. 5545-5552, 2014.

[18] S. Abbasbandy, "The application of homotopy analysis method to nonlinear equations arising in heat transfer," Physics Letters A, vol. 360, no. 1, pp. 109-113, 2006.

[19] A. Atangana, "On the new fractional derivative and application to nonlinear Fisher's reaction-diffusion equation," Applied Mathematics and Computation, vol. 273, pp. 948-956, 2016.

[20] M. Usman, M. Hamid, and M. Liu, "Novel operational matrices-based finite difference/spectral algorithm for a class of time-fractional Burger equation in multidimensions," Chaos, Solitons and Fractals, vol. 144, article 110701, 2021. 
[21] S. S. Zeid, "Approximation methods for solving fractional equations," Chaos, Solitons and Fractals, vol. 125, pp. 171193, 2019.

[22] M. Hamid, M. Usman, R. U. Haq, and W. Wang, "A Chelyshkov polynomial based algorithm to analyze the transport dynamics and anomalous diffusion in fractional model," Physica A: Statistical Mechanics and its Applications, vol. 551, article 124227, 2020.

[23] A. El-Ajou, M. Al-Smadi, M. N. Oqielat, S. M. Momani, and S. Hadid, "Smooth expansion to solve high-order linear conformable fractional PDEs via residual power series method: applications to physical and engineering equations," Ain Shams Engineering Journal, vol. 11, no. 4, pp. 1243-1254, 2020.

[24] A. Arafa and G. Elmahdy, "Application of residual power series method to fractional coupled physical equations arising in fluids flow," International Journal of Differential Equations, vol. 2018, Article ID 7692849, 10 pages, 2018.

[25] M. Alquran, "Analytical solutions of fractional foam drainage equation by residual power series method," The Mathematical Scientist, vol. 8, no. 4, pp. 153-160, 2014.

[26] Marwan Alquran, "Analytical solution of time-fractional twocomponent evolutionary system of order 2 by residual power series method," Journal of Applied Analysis \& Computation, vol. 5, no. 4, pp. 589-599, 2015.

[27] R. M. Jena and S. Chakraverty, "Residual power series method for solving time-fractional model of vibration equation of large membranes," Journal of Applied and Computational Mechanics, vol. 5, no. 4, pp. 603-615, 2019.

[28] E. M. Khalil, Z. Korpinar, and M. Inc, "Investigation of numerical solutions of fractional generalized reguralized long wave equations by least squares-residual power series method," Physica Scripta, vol. 96, no. 9, article 094005, 2021.

[29] A. el-Ajou, O. A. Arqub, and S. Momani, "Approximate analytical solution of the nonlinear fractional KdV-Burgers equation: a new iterative algorithm," Journal of Computational Physics, vol. 293, pp. 81-95, 2015.

[30] H. M. Jaradat, S. Al-Shara, Q. J. A. Khan, M. Alquran, and K. Al-Khaled, "Analytical solution of time fractional Drinfeld-Sokolov-Wilson system using residual power series method," International Journal of Applied Mathematics, vol. 46, pp. 64-70, 2016.

[31] F. Xu, Y. Gao, X. Yang, and H. Zhang, "Construction of fractional power series solutions to fractional boussinesq equations using residual power series method," Mathematical Problems in Engineering, vol. 2016, Article ID 5492535, 15 pages, 2016.

[32] A. El-Ajou, O. Abu Arqub, S. Momani, D. Baleanu, and A. Alsaedi, "A novel expansion iterative method for solving linear partial differential equations of fractional order," Applied Mathematics and Computation, vol. 257, pp. 119133, 2015.

[33] M. Aylin Bayrak and A. Demir, "A new approach for spacetime fractional partial differential equations by residual power series method," Applied Mathematics and Computation, vol. 336, pp. 215-230, 2018.

[34] A. A. M. Arafa, "A new algorithm of residual power series (RPS) technique," International Journal of Applied and Computational Mathematics, vol. 6, no. 3, p. 62, 2020. 